\title{
3 Research Suare \\ Poor Physical Capacity in Bronchiectasis Patients Is Correlated with Poor Quality of Life
}

Jarkko Mäntylä ( $\square$ jarkko.mantyla@helsinki.fi )

Helsingin Yliopisto https://orcid.org/0000-0002-9640-4872

Witold Mazur

Helsingin ja Uudenmaan sairaanhoitopiiri

Tanja Törölä

Helsingin Yliopisto

Paula Bergman

Helsingin Yliopisto

Paula Kauppi

Helsingin ja Uudenmaan sairaanhoitopiiri

\section{Research article}

Keywords: bronchiectasis severity index, quality of life, extensive disease, exacerbation

Posted Date: June 10th, 2020

DOI: https://doi.org/10.21203/rs.3.rs-32115/v1

License: (c) (1) This work is licensed under a Creative Commons Attribution 4.0 International License.

Read Full License 


\section{Abstract}

Background: Bronchiectasis (BE) is an increasing burden on healthcare systems, and there is an urgent need for improved treatments, better clinical care and for clinical and translational research on this condition. Reducing the number of exacerbations is one of the key goals in BE management. Patients who suffer from frequent exacerbations are likely to experience more exacerbations, which have negative effects on quality of life (QoL), healthcare utilisation and mortality. Thus, we aimed to investigate the risk factors associated with quality of life (QoL) in a cohort of Finnish non-cystic fibrosis BE patients.

Methods: A cross-sectional study is part of the EMBARC. Recruitment occurred between August 2016 and March 2018 from three clinics in Helsinki University Central Hospital, Finland. The study included 95 adult patients (mean age was 69 (SD \pm 13 ) years). Seventy nine percent were women. A disease-specific quality of life-bronchiectasis (QoL-B) questionnaire was applied, and scores in the lowest quarter (24\%) indicate poor QoL. The bronchiectasis severity index (BSI) and FACED score were used. The severity of dyspnoea was examined using the modified Medical Research Council (mMRC) dyspnoea scale.

Results: Almost all (82\%) presented with chronic sputum production and exacerbation, with a median rate of 1.7 (SD \pm 1.6 ). Exacerbations (OR 1.7), frequent exacerbations (OR 4.9, $p<0.01$ ), high BSI score (OR $1.3, p<0.01)$ and extensive disease (OR 3.7, $p=0.05)$ were predictive of poor QoL. Frequent exacerbations were associated with bronchial bacterial colonisation, low forced expiratory volume in one second (FEV1) and radiological disease severity. Based on the BSI, $34.1 \%$ of our cohort had severe disease, whereas $11.6 \%$ were classified as severe according to the FACED score. The mMRC dyspnoea score $(r=-0.57)$ and BSI $(r=-0.60)$ were negatively correlated with physical domain in QoL-B questionnaire.

Conclusion: Frequent exacerbations, radiological disease severity and high BSI score were predictive of poor QoL. Reduced physical capacity was correlated with dyspnoea and severity of disease. Interventions to reduce bacterial colonisation and to maintain physical functioning should be used to minimize exacerbations and to improve QoL.

Study registration: University of Helsinki, faculty of medicine; 148/16.08.2017; Registered 16 August 2017; https://researchportal.helsinki.fi/

\section{Background}

Irreversible destruction and enlargement of bronchi is a typical feature of bronchiectasis (BE). Chronic intrabronchial neutrophilic inflammation and potential bacterial colonisation cause chronic daily airway symptoms such as excessive sputum production and recurrent airway infections. The impact of morbidity burden and diminished quality of life $(\mathrm{QoL})$ from this vicious cycle is obviously unfavourable. $B E$ is a long-term condition with no curative treatment, for which the aim is to prevent exacerbations and further deterioration of BE and maintain or improve QoL. Despite extensive research, the aetiology of BE remains unknown. 
Characterisation of the disease has been challenging in the past due to a lack of validated tools. For this reason, tools to optimise individual treatments and improve clinical care are urgently needed, in addition to better tools to assess the effect of treatment and disease progression. Due to the heterogeneity of $B E$, only a limited number of effective treatments and evidence-based recommendations are available. However, it is important to assess the subgroups that would gain the most benefit from a specific intervention.

Commonly used measures for different lung diseases have serious limitations in clinical trials [1]. For example, spirometry provides a good assessment of lung function but does not assess the specific health status or severity of the disease [2]. Similarly, radiographic examinations, such as high resolution computed tomography (HRCT) scans, have been used to describe the severity of $B E$, but the findings do not correlate well with the clinical features of the disease [3]. Tests to estimate dyspnoea and physical capability (e.g., 6-minutes walking test) are useful and standardised in routine praxis. However, a study by Lee et al. [2] demonstrated that this test was not appropriate to describe the severity of BE.

The first of the questionnaires developed for respiratory patients, especially those with BE, was the St George's Respiratory Questionnaire (SGRQ), which was followed by a number of disease-specific questionnaires for chronic obstructive pulmonary disease (COPD) and pulmonary fibrosis. Wilson et al. [4] validated the SGRQ for BE in 1997 and showed a good correlation of the SGRQ score with disease severity. Another patient-reported questionnaire for BE, the Leicester Cough Questionnaire, inadequately covers the respiratory symptoms [5].

Modern clinical assessment tools for BE have only been used in the past few years. Chalmers et al. [6] validated the bronchiectasis severity index (BSI) in 2014, and Martinez-Garcia et al. [7] established the FACED score in the same year. These tools are exceptional for the assessment of symptomatic or frequently exacerbated patients with severe disease, enabling focused and intensive treatment. Nowadays, both the BSI and FACED have significant roles in assessing the severity of BE.

Quittner et al. [8] validated the disease-specific quality of life questionnaire-bronchiectasis (QoL-B) version 3.0 in 2014, and confirmed its applicability for use in clinical trials and routine clinical practice. They assumed the relevance for evaluating BE as a whole.

In the current study, we studied the risk factors for poor QoL in a Finnish BE cohort using clinical assessment tools.

\section{Methods}

\section{Participants}

Altogether, 95 of 205 non-cystic fibrosis BE patients were included in this cross-sectional study, which focused on characterising the cohort. Patients were recruited between August 2016 and March 2018 from the Helsinki University Hospital (HUH) district. The inclusion criteria for patients were as follows: 1) 
doctor-patient relationship in $\mathrm{HUH}$; 2) over 18 years of age; 3) high-resolution computed tomography (HRCT) used to diagnose BE; and 4) non-cystic fibrosis BE. Details of this cohort have been reported previously [9].

\section{Quality of life in bronchiectasis questionnaire}

The QoL-B (version 3.0) is a multidimensional disease-specific questionnaire for patients with BE. Patient's self-report their current condition over 37 questions, which are categorised into eight different domains (respiratory symptoms, physical activity, role, emotional and social functioning, vitality, health perceptions and treatment burden). The score for each domain ranges from 0 to 100, with higher scores representing fewer symptoms or better functioning. The QoL-B has been reported to give reliable and consistent results if re-tested and has high convergent validity [8][10]. The QoL-B has been translated into Finnish, which was the version applied in the current study.

\section{Modified Medical Research Council scale}

In patients with respiratory diseases, the modified Medical Research Council (mMRC) dyspnoea scale is a reliable tool to assess functional disability due to dyspnoea. Dyspnoea only on strenuous exercise on level ground is graded 0 points, shortness of breath when hurrying on level ground or walking up a slight hill is graded 1 point, walking slower than people of the same age on level ground due to breathlessness or the need to stop to catch breath when walking at their own pace is graded 2 points, stopping for breath after walking $100 \mathrm{~m}$ or after a few minutes on level ground is graded 3 points, and breathless when dressing is graded 4 points. Higher grades on the mMRC scale indicate a higher severity of BE [11][12].

\section{Bronchiectasis Severity Index}

The Bronchiectasis Severity Index (BSI) is a composite disease-specific prognostic index that was developed to aid clinical decision-making related to BE. Specifically, the BSI was developed to predict mortality, severe exacerbations, frequency of exacerbations and QoL [6]. The BSI combines the clinical, radiological and microbiological features of $\mathrm{BE}$, and includes the following parameters: body mass index (BMI), forced expiratory volume in one second (FEV1; \% of predicted), previous hospital admissions, number of exacerbations in the previous year, mMRC breathlessness score, pseudomonas colonisation or colonisation with other microorganisms, and radiological severity. The grading system of the BSI is mild (0-4 points), moderate (5-8 points) and severe (9 or more points) [6].

\section{FACED score}

The FACED score is another disease-specific prognostic index that aims to assess the probability of allcause mortality over 5 or 15 years of follow-up [6][7][13]. FACED assesses different factors related to BE including lung function (FEV1), age, pulmonary bacterial colonisation, number of lobes affected by BE, and dyspnoea. FACED scoring is graded as mild (0-2 points), moderate (3-4 points) or severe (5-7 points). 


\section{Patient and Public Involvement:}

This is the part of EMBARC study. The data were collected into the EMBARC database anonymously. Patients or the public were not involved in the design, conduct, or reporting, of our research.

Dissemination of our research will be offered through the Finnish Organisation for Respiratory Health and through the Finnish Allergy, Skin and Asthma Federation.

\section{Statistical analyses}

Independent samples t-test and Mann-Whitney U test were used to compare means and mean ranks, respectively. Proportions were compared by two-tailed z-test. Risk factors for poor quality of life in BE were analysed by logistic regression analysis, with age, BMI, FEV1 (\%) and FVC (\%) considered as continuous variables. Statistical analyses were performed with Statistical for Social Sciences (SPSS) program, version 22 (IBM corporation, Armonk NY, USA).

\section{Ethical approval}

The ethics committee of HUH approved the study (registration number 214/13/03/01/2016).

\section{Results}

The overall QoL results (median values) for different symptom domains were 60 (SD \pm 31.9 ) for physical functioning, $60( \pm 27.9)$ for role, $50( \pm 20.5)$ for vitality, $75( \pm 22.2)$ for emotional functioning, $58( \pm 24.4)$ for social functioning, $77.8( \pm 24.8)$ for treatment burden, $41.7( \pm 21.1)$ for health perceptions, $57( \pm 19.7)$ for respiratory symptoms and $450( \pm 151.8)$ for all domains combined (where 0 was the minimum and 800 the maximum). A score in the lowest quarter of the scale, less than $25 \%$, represented a poor QoL.

In the total BE cohort, dyspnoea assessed by $\mathrm{mMRC}$ had a mean value of $1.72( \pm 1.1)$ and median value of $2( \pm 1.1)$, with $18.9 \%$ of patients having a mMRC score of 3 and $6.3 \%$ having a score of 4 .

Overall, the BE cohort had a mean BSI value of 7.7 (SD 4.2) and median value of 7 (SD 2.0). Of the BE patients, $22 \%$ had mild disease, $44 \%$ had moderate disease and $34.1 \%$ had severe disease.

When BE severity was estimated according to the FACED index, $42.1 \%$ of patients had mild BE, $46.3 \%$ had moderate $B E$ and $11.6 \%$ had severe BE. The mean score was $2.6( \pm 1.5)$ and the median was $3.0( \pm 1.5)$.

The BE patients with a poor QoL had lower lung function (FEV1 $84.3 \pm 31.4 \%$ ) than those with preserved QoL (Table 1). Further, patients with poor QoL had more severe dyspnoea (mMRC mean scores of 2.4 vs. 1.5) and more severe BE disease (BSI of 10.5 vs. 6.9), a greater number of exacerbations (four vs. two over the past 12 months), extensive disease, and more frequent bacterial colonisation and cystic changes. 
The mMRC dyspnoea score had a moderate negative correlation with physical domain $(r=-5.72, p<$ 0.001) in the QoL questionnaire (Table 2). Similarly, BSI had a moderate negative correlation with physical domain $(r=-5.96, p<0.001)$ in the QoL questionnaire (Table 2).

Those patients with several frequent yearly exacerbations of BE had poorer lung function (FEV1 of 79\% vs. $93 \%$ ), were more symptomatic with dyspnoea, had more severe disease (BSI of $5 \pm 3.2$ vs. $10 \pm 3.9$ ), more frequent bacterial colonisation ( $53.8 \%$ vs. $19.6 \%$ ) and cystic BE changes, and more often had asthma as the aetiology for $\mathrm{BE}(30 \%$ vs. $23 \%$ ) than those with fewer exacerbations (Table 3 ).

When analysing the risk factors for poor QoL, exacerbations, BSI, extensive disease and frequent yearly exacerbations were found to be important. When the analysis was adjusted for age and gender, exacerbations (OR 1.7), extensive disease (OR 3.7) and frequent yearly exacerbations (OR 4.9) increased the risk for a poor QoL (Table 4).

\section{Discussion}

Exacerbations of BE and extensive disease significantly increase the risk of a poor QoL. In particular, recurrent yearly exacerbations were found to increase the risk of poor QoL by five-fold. In this study, both effort tolerance (mMRC) and disease severity (BSI) were negatively correlated with physical domain which is formed of the questions assessing physical capability associated with BE in the quality of life questionnaire. However, in clinical practice, the mMRC dyspnoea score is simpler and easier to use than the BSI [6][12]. The BSI is a complex index that requires lung function parameters, radiological features, exacerbation, hospitalisation and microbiological findings to calculate the score [6]. On the other hand, the mMRC is a four-point grading system for self-reported dyspnoea in everyday life [12]. Although BSI has been shown to accurately predict hospitalisations, exacerbations and mortality of non-cystic fibrosis BE patients, it is more challenging to use and is therefore better suited to scientific purposes than clinical practice [6][14]. The mMRC and QoL scores have been used to demonstrate the effect of new treatment options for BE [14].

Health (median score of 41.7), vitality (median score of 50) and respiration (median score of 57) were the domains with the poorest self-reported results in the QoL questionnaire. It has previously been demonstrated that these scores are stable if measured during a stable phase of $\mathrm{BE}$, and that they can be used to differentiate between mild, moderate and severe BE patients [7]. However, in a 2-year follow-up study of $19 \mathrm{BE}$ patients by Magge et al. [15], physical functioning, role functioning and health perceptions were shown to improve with treatment at a specialised care center. These results are similar to ours for health perceptions (mean score 41.4) and vitality (mean score 45.6), but not for respiration (mean score 62.5).

Our finding of significantly reduced vitality score and $\mathrm{mMRC}$ score correlating with the physical domain is in accordance with previous studies that show reduced physical activity of BE patients compared to gender- and age-matched individuals without BE [16]. In our study, the mMRC scale offered practical information on the physical functioning of patients. 
The identification of patients at high risk for exacerbations may be valuable to guide clinical decision making with regard to factors such as the frequency and intensity of follow-up and the use of long-term antibiotic therapy. The scoring systems used to assess the severity of BE gave different results for severe disease, as $34.1 \%$ of patients were estimated to have severe BE with BSI scoring while only $11.6 \%$ of patients were classified as having severe BE with FACED grading. Similar results were described by Visser et al. [17] in their Australian cohort, as $58 \%$ of the cohort had severe disease according to the BSI, whereas $17 \%$ had FACED-defined severe disease. Our results are also in good agreement with the observations of a large multidimensional severity assessment conducted by McDonnell et al. [14], who reported that the BSI is superior to FACED in predicting overall clinically important disease-related outcomes. The BSI includes parameters for previous hospital admissions and the number of exacerbations in the previous year, whereas FACED includes a parameter for age [6][7][13]. Although, they have been shown to have similar capacities for predicting long-term mortality, in our cross-sectional study more patients were classified as having severe BE by BSI scoring than by FACED scoring [13]. This highlights the importance of patients' previous history of exacerbations.

When phenotyping different BE patients, BE caused by connective tissue disease has been associated with a poor prognosis and rapid disease progression [18]. Immunodeficiency, COPD and allergic bronchopulmonary aspergillosis (ABPA) have been associated with recurrent exacerbations [18]. In the current study, we found poor QoL regardless of background aetiology in BE patients with exacerbations, and especially in those with frequent exacerbations. Furthermore, colonisation of the bronchi with proteobacteria Pseudomonas aeruginosa or Haemophilus influenza is associated with chronic neutrophilic inflammation and exacerbations [18].

Anti-IL5 and anti-IL5R therapies have been suggested as an add-on therapy for BE [19]. A study that included BE patients with severe asthma, ABPA, idiopathic BE, combined asthma/COPD, EGPA and PCD reported an increase in FEV1 and QoL, as well as a reduction in exacerbations and mMRC scores [19].

Terpstra et al. [20] reported significantly worse QoL in BE patients with COPD than in other BE patients. In their study population of 200 BE patients (aged 69.5 years), the mean number of exacerbations was one per year, with zero hospitalisations and a mean mMRC score of 2.68 (SD 1.09). The mean mMRC score in our population was 2.0, but there was a median of two exacerbations per year. Previously, BSI was proposed as a tool for scoring the severity of BE and FACED as a tool for predicting mortality [20][21].

The limitations of the current study include the relatively small size of the cohort and the cross-sectional setting of the QoL analysis. The small sample size is due to the limited number of subjects recruited in the original study. A strength of the study is that there were no missing data for QoL questions and mMRC scale results.

An earlier study found that previous severe exacerbations (OR 2.6) or an exacerbation in the past 12 months (OR from 1.38 to 3.90) is predictive of future exacerbations [6]. We found that exacerbations (OR 1.7), frequent yearly exacerbations (OR 4.9) or extensive disease (OR 3.7) increased the risk of poor QoL (Table 4) in the analysis adjusted for age and gender. 
Similarly, diminished lung function (analysed as FEV1) has been reported to be a risk factor for future exacerbations [6]. Although those patients with poor QoL had reduced lung function (FEV1 84\%) than the others (FEV1 89\%), we did not identify lung function as a risk factor for future exacerbations [6]. This may be due to the relatively small study population, together with the relatively well-preserved lung function in our study population.

When estimating the effect of exacerbations and frequent exacerbations on poor QoL, it should be noted that in the study by Brill et al. [22], the median duration of an exacerbation was 16 days, and $16 \%$ of patients had at least one exacerbation with a duration of more than 35 days. Thus, the association of poor QoL with exacerbations is crucial. Despite best practice guidelines, exacerbation will occur at some point, so the secondary treatment goals are to prevent deterioration of lung function and BE radiologic findings and to maintain a good QoL. This should be considered when assessing which BE patients benefit from follow-up at a specialised care center [15]. The current guidelines focus on recommending special care for those with three or more yearly exacerbations and for those on long-term antimicrobial therapy [23][24].

\section{Conclusions}

We found that exacerbations, especially frequent exacerbations, increased the risk of poor QoL in patients with BE. Neither comorbidities nor the aetiology of BE appeared to affect the QoL. In addition, more extensive disease or greater severity of BE increased the risk of poor QoL. Furthermore, the mMRC score for dyspnoea and BSI for severity of BE showed the strongest (negative) correlations with physical functioning in BE. When looking for strategies to improve QoL in BE, the focus should be on preventing exacerbations and supporting physical fitness to reduce exercise-induced dyspnoea and support patients' ability to cope in daily life activities and also when at an advanced disease status

\section{Strengths And Limitations Of The Study}

- Strength of this study is the multifaceted perspective to symptoms by comparing QoL questionnaire with $\mathrm{mMRC}, \mathrm{BSI}$ and FACED score results.

- Further, there were no missing data for QoL questions or for mMRC results and thus the results are valid for this study population.

- Limitations of this study are a cross-sectional setting of the analysis and the relatively small number of study participants which is reflective of prevalence of BE but also the relatively small number of population in Finland and in Helsinki and Uusimaa Hospital area.

\section{Abbreviations}

ABPA = Allergic bronchopulmonary aspergillosis

$\mathrm{BE}=$ Bronchiectasis 
$\mathrm{BMI}=$ Body mass index

$\mathrm{BSI}=$ Bronchiectasis severity index

COPD $=$ Chronic obstructive pulmonary disease

EGPA $=$ Eosinophilic granulomatosis with polyangiitis

FEV1 = Forced expiratory volume in one second

HRCT = High resolution computed tomography

HUH = Helsinki University Hospital

mMRC $=$ Modified Medical Research Council

PCD = Primary ciliary dyskinesia

QoL = Quality of life

QoL-B = Disease-specific quality of life questionnaire-bronchiectasis

SGRQ = St George's Respiratory Questionnaire

\section{Declarations}

Ethics approval and consent to participate: The ethics committee of HUH approved the study (registration number 214/13/03/01/2016). All patients provided written informed consent.

Consent for Publication: Not applicable

Availability of data and material: This is part of the EMBARC study and data can be obtained from EMBARC register or from the authors by an approved application.

Competing interests: In relation to this study, the authors declare that there are no conflicts of interest.

Funding: This study was supported by funding of the Foundation of the Finnish Anti-Tuberculosis Association, The Finnish Research Foundation of the Pulmonary Diseases and University of Helsinki and Helsinki University Hospital, Heart and Lung Center, Department of Respiratory Diseases, Helsinki, Finland

The funders of the study had no role in the study design, data collection, data analysis, data interpretation or writing of the report.

Authors' contributions: PK was responsible for the design, analysis, interpretation, drafting and finalizing of the manuscript. WM was involved in the analysis, interpretation, drafting and finalizing of the manuscript. JM was responsible for the data collection, analysis, interpretation, drafting and finalizing of the manuscript. TT was involved in the data collection, drafting and finalizing of the manuscript. PB 
provided statistical assistance and was involved in finalizing of the manuscript. All authors read and approved the final manuscript.

Acknowledgements: The authors would like to acknowledge all the patients who participated in the study.

\section{References}

1. Smith MP, Hill AT. Evaluating Success of Therapy for Bronchiectasis. What End Points to Use? Clin Chest Med. 2012;33:329-49. doi:10.1016/j.ccm.2012.03.001.

2. Lee AL, Button BM, Ellis S, Stirling R, Wilson JW, Holland AE, et al. Clinical determinants of the 6Minute Walk Test in bronchiectasis. Respir Med. 2009;103:780-5.

3. Eshed I, Minski I, Katz R, Jones PW, Priel IE. Bronchiectasis: correlation of high-resolution CT findings with health-related quality of life. Clin Radiol. 2007.

4. Wilson CB, Jones PW, O'Leary CJ, Cole PJ, Wilson R. Validation of the St. George's respiratory questionnaire in bronchiectasis. Am J Respir Crit Care Med. 1997;156 2 I:536-41.

5. Murray MP, Turnbull K, MacQuarrie S, Pentland JL, Hill AT. Validation of the Leicester Cough Questionnaire in non-cystic fibrosis bronchiectasis. Eur Respir J. 2009;34:125-31.

6. Chalmers JD, Goeminne P, Aliberti S, Mcdonnell MJ, Lonni S, Davidson J, et al. The Bronchiectasis Severity Index An International Derivation and Validation Study. 2014;189:576-85.

7. Martínez-García MÁ, de Gracia J, Vendrell Relat M, Girón R-M, Máiz Carro L, de la Rosa Carrillo D, et al. Multidimensional approach to non-cystic fibrosis bronchiectasis: the FACED score. Eur Respir J. 2014;43:1357-67. doi:10.1183/09031936.00026313.

8. Quittner AL, Marciel KK, Salathe MA, O'Donnell AE, Gotfried MH, llowite JS, et al. A preliminary quality of life questionnaire-bronchiectasis: A patient-reported outcome measure for bronchiectasis. Chest. 2014.

9. Mäntylä J, Mazur W, Törölä T, Bergman P, Saarinen T, Kauppi P. Asthma as aetiology of bronchiectasis in Finland. Respir Med. 2019;152:105-11.

10. Olveira C, Olveira G, Espildora F, Giron RM, Muñoz G, Quittner AL, et al. Validation of a Quality of Life Questionnaire for Bronchiectasis: Psychometric analyses of the Spanish QOL-B-V3.0. Qual Life Res. 2014.

11. Mahler DA, Welss CK. Evaluation of Clinical Methods for Rating. Chest. 1988;93:580-6. doi:10.1378/chest.93.3.580.

12. Bestall JC, Paul EA, Garrod R, Garnham R, Jones PW, Wedzicha JA. Usefulness of the Medical Research Council (MRC) dyspnoea scale as a measure of disability in patients with chronic obstructive pulmonary disease. Thorax. 1999;54:581-6.

13. Ellis HC, Cowman S, Fernandes M, Wilson R, Loebinger MR. Predicting mortality in bronchiectasis using bronchiectasis severity index and FACED scores: a 19-year cohort study. :482-9. doi:10.1183/13993003.01312-2015. 
14. McDonnell MJ, Aliberti S, Goeminne PC, Dimakou K, Zucchetti SC, Davidson J, et al. Multidimensional severity assessment in bronchiectasis: an analysis of seven European cohorts. Thorax. 2016;71:1110-8. doi:10.1136/thoraxjnl-2016-208481.

15. Magge A, Ashraf S, Quittner AL, Metersky ML. Quality of life in patients with bronchiectasis: a 2-year longitudinal study. Ann Transl Med. 2019;7:334-334.

16. Cakmak A, Inal-Ince D, Sonbahar-Ulu H, Bozdemir-Ozel C, Ozalp O, Calik-Kutukcu E, et al. Physical activity of patients with bronchiectasis compared with healthy counterparts: A cross-sectional study. Heart Lung. 2019. doi:10.1016/j.hrtlng.2019.09.004.

17. Visser SK, Bye PTP, Fox GJ, Burr LD, Chang AB, Holmes-liew C, et al. Australian adults with bronchiectasis: The fi rst report from the Australian Bronchiectasis Registry. 2019;155 February:97103.

18. Flume PA, Chalmers JD, Olivier KN. Advances in bronchiectasis: endotyping, genetics, microbiome, and disease heterogeneity. Lancet. 2018;392:880-90. doi:10.1016/S0140-6736(18)31767-7.

19. Rademacher J, Konwert S, Fuge J, Dettmer S, Welte T, Ringshausen FC. Anti-IL5 and anti-IL5Ra therapy for clinically significant bronchiectasis with eosinophilic endotype: a case series. Eur Respir J. 2019;:1901333.

20. Terpstra LC, Biesenbeek S, Altenburg J, Boersma WG. Aetiology and disease severity are among the determinants of quality of life in bronchiectasis. Clin Respir J. 2019;13:521-9. doi:10.1111/crj.13054.

21. Mendes MA, Chalmers JD. Predicting outcomes in bronchiectasis. Pulmonology. 2018;24:146-8.

22. Brill SE, Patel ARC, Singh R, Mackay AJ, Brown JS, Hurst JR. Lung function, symptoms and inflammation during exacerbations of non-cystic fibrosis bronchiectasis: a prospective observational cohort study. Respir Res. 2015;16:16. doi:10.1186/s12931-015-0167-9.

23. Hill AT, Sullivan AL, Chalmers JD, De Soyza A, Stuart Elborn J, Andres Floto R, et al. British thoracic society guideline for bronchiectasis in adults. Thorax. 2019;74.

24. Polverino E, Goeminne PC, McDonnell MJ, Aliberti S, Marshall SE, Loebinger MR, et al. European Respiratory Society guidelines for the management of adult bronchiectasis. European Respiratory Journal. 2017;50.

\section{Tables}


Table 1. Clinical characteristics of the bronchiectasis patients with poor quality of life and those without poor quality of life

\begin{tabular}{|c|c|c|c|}
\hline & Poor QoL & Others & $p$-value \\
\hline $\mathrm{N}=92$, Missing $n=3$ & $n=23 / 24 \%$ & $n=69 / 73 \%$ & \\
\hline Age, median & 72 & 71 & 0.18 \\
\hline Gender, female & 95.7 & 75.4 & 0.03 \\
\hline Ever-smokers (\%) & 21.7 & 36.2 & 0.2 \\
\hline BMI, mean $( \pm S D)$ & $26.8(5.2)$ & $25.7(5.5)$ & 0.37 \\
\hline FEV1\%, mean $( \pm S D)$ & $84.3(31.4)$ & $88.9(21.4)$ & 0.22 \\
\hline mMRC, mean $( \pm S D)$ & $2.43(0.896)$ & 1.49 (1.093) & $<0.01$ \\
\hline $\mathrm{BSI}( \pm \mathrm{SD})$ & $10.5(3.8)$ & $6.9(4.0)$ & $<0.01$ \\
\hline FACED, mean $( \pm S D)$ & $3.1(1.3)$ & $2.5(1.5)$ & 0.05 \\
\hline Exacerbations (median) & 4 & 2 & $<0.01$ \\
\hline Extensive disease $(\%)$ & 87 & 62.3 & 0.03 \\
\hline Ever had bacterial colonisation (\%) & 43.5 & 30.4 & 0.25 \\
\hline Cystic changes (\%) & 13 & 5.8 & 0.26 \\
\hline \multicolumn{4}{|l|}{ Aetiology (\%) } \\
\hline Idiopathic & 21.7 & 47.8 & 0.03 \\
\hline Asthma & 39.1 & 23.2 & 0.14 \\
\hline Postinfectious & 8.7 & 11.6 & 0.7 \\
\hline Other & 30.4 & 17.4 & 0.18 \\
\hline
\end{tabular}

BMI = Body Mass Index $(\mathrm{kg} / \mathrm{ml}) ; \quad F E V 1 \%=F E V 1 / F V C=$ Forced expiratory volume in one second / Forced vital capacity; $Q o L=$ Quality of life; $m M R C=$ Modified medical research council; BSI = Bronchiectasis severity index

Table 2. Correlation of mMRC score and BSI with different symptom domains

\begin{tabular}{lccc|ccc} 
Domain & mMRC & $p$-value & $\mathrm{N}$ & $\mathrm{BSI}$ & $p$-value & $\mathrm{N}$ \\
\hline Physical & -0.572 & 0.000 & 92 & -0.596 & 0.000 & 88 \\
Role & -0.457 & 0.000 & 92 & -0.470 & 0.000 & 88 \\
\hline Vitality & -0.342 & 0.001 & 92 & -0.343 & 0.001 & 88 \\
\hline Emotion & -0.254 & 0.015 & 92 & -0.343 & 0.001 & 88 \\
\hline Social & -0.227 & 0.029 & 92 & -0.243 & 0.023 & 88 \\
Treatment burden & -0.210 & 0.045 & 92 & -0.406 & 0.000 & 88 \\
\hline Health & -0.322 & 0.002 & 92 & -0.446 & 0.000 & 88 \\
\hline Respiration & -0.294 & 0.004 & 92 & -0.395 & 0.000 & 88 \\
\hline
\end{tabular}

$m M R C=$ Modified medical research council $; B S I=$ Bronchiectasis severity index 
Table 3. Characteristics of all the BE patients, BE patients with 0-2 exacerbations per year and BE patients with $>2$ exacerbations per year

\begin{tabular}{|c|c|c|c|c|}
\hline & $\begin{array}{l}\text { Full } \\
\text { cohort }\end{array}$ & $\begin{array}{c}\text { Exacerbation 0- } \\
2 / y\end{array}$ & $\begin{array}{c}\text { Exacerbation }> \\
2 / y\end{array}$ & $p$-value \\
\hline $\mathrm{N}$ & 95 & 56 & 39 & \\
\hline Gender, female (\%) & 75 & 80 & 77 & 0.68 \\
\hline Age, median (IQR) & 71 & $71(65.5-76)$ & $72(58-78)$ & 0.79 \\
\hline BMI, median (IQR) & 26 & $24.9(21.5-30.6)$ & $26.4(22.5-29.5)$ & 0.31 \\
\hline Ever-smokers (\%) & 33.7 & 35.8 & 30.8 & 0.78 \\
\hline FEV1\%, mean ( \pm SD) & 87 & $93.2(23.8)$ & $79.1(22.6)$ & 0.01 \\
\hline Exacerbations, median & 2 & 1 & 4 & $<0.01$ \\
\hline Extensive disease (\%) & 68.4 & 60.7 & 79.5 & 0.05 \\
\hline $\begin{array}{l}\text { Ever had bacterial colonisation } \\
\text { (\%) }\end{array}$ & 33.7 & 19.6 & 53.8 & 0.06 \\
\hline Cystic changes (\%) & 9 & 7.1 & 12.8 & 0.78 \\
\hline Poor QoL (\%) & 24.2 & 14.3 & 38.5 & 0.23 \\
\hline mMRC mean $( \pm S D)$ & 2 & $1.5(1.1)$ & $2.1(1.0)$ & 0.03 \\
\hline BSI mean $( \pm S D)$ & 7 & $5.9(3.2)$ & $10.5(3.9)$ & $<0.01$ \\
\hline FACED, mean $( \pm S D)$ & 2.1 & $2.4(1.5)$ & $2.9(1.5)$ & 0.13 \\
\hline \multicolumn{5}{|l|}{ Aetiology } \\
\hline Idiopathic (\%) & 41 & 46.4 & 38.5 & 0.62 \\
\hline Asthma (\%) & 26 & 23.2 & 30.8 & 0.67 \\
\hline Postinfectious (\%) & 11 & 12.5 & 7.7 & 0.82 \\
\hline Other $(\%)$ & 22 & 17.9 & 23.1 & 0.78 \\
\hline
\end{tabular}

$B M I=$ Body Mass Index $(\mathrm{kg} / \mathrm{m} I) ; F E V 1 \%=$ FEV1/FVC = Forced expiratory volume in one second $/$ Forced vital capacity;

$Q o L=$ Quality of life $m M R C=$ Modified medical research council; BSI = Bronchiectasis severity index 
Table 4. Risk factors for poor quality of life in bronchiectasis

Adjusted Age and Gender

\begin{tabular}{lcccc} 
& $p$-value & Ext(B) & 95\% C.I.for EXP(B) \\
\hline Gender (women) & 0.06 & & & \\
\hline Age & 0.21 & & & \\
\hline Cardiovascular disease & 0.44 & 1.571 & 0.5 & 4.93 \\
\hline Psychiatric disisease & 0.07 & 0.224 & 0.05 & 1.12 \\
\hline Connective tissue disease & 0.21 & 0.974 & 0.93 & 1.02 \\
\hline BMI & 0.3 & 0.951 & 0.87 & 1.05 \\
\hline FEV1\% & 0.27 & 1.013 & 0.99 & 1.04 \\
\hline Asthma dg & 0.28 & 0.532 & 0.17 & 1.66 \\
\hline Asthma aetiology & 0.18 & 0.979 & 0.94 & 1.4 \\
\hline Exacerbations & 0.00 & 1.718 & 1.29 & 2.3 \\
\hline FACED & 0.16 & 1.354 & 0.89 & 2.05 \\
\hline BSI & $<0.01$ & 1.258 & 1.09 & 1.46 \\
\hline mMRC & $<0.01$ & 0.427 & 0.25 & 0.73 \\
\hline Extensive disease & 0.05 & 3.687 & 0.97 & 14.02 \\
\hline Have ever bacterial colonisation & 0.21 & 0.518 & 0.19 & 1.43 \\
\hline Frequent exacerbator $>2 / y$ & $<0.01$ & 4.946 & 1.69 & 14.44 \\
\hline
\end{tabular}

$B M I=$ Body Mass Index $(\mathrm{kg} / \mathrm{ml}) ; \mathrm{FEV1} \%=$ FEV1 $/ F V C=$ Forced expiratory volume in one second $/$ Forced vital capacity; $Q o L=$ Quality of life; BSI = Bronchiectasis severity index; $m M R C=$ Modified medical research council 